\title{
Quantitative Clinical Cardiac Magnetic Resonance Imaging Quantitative klinische Herz-Magnetresonanztomografie
}

Authors

Ursula Reiter ${ }^{1}$, Clemens Reiter ${ }^{1}$, Corina Kräuter ${ }^{1,2}$, Volha Nizhnikava1, 3, Michael H. Fuchsjäger ${ }^{1}$, Gert Reiter ${ }^{1,4}$

Affiliations

1 Radiology, Medical University of Graz, Austria

2 Institute of Medical Engineering, Graz University of Technology, Faculty of Computer Science and Biomedical Engineering, Graz, Austria

3 Radiology, Respublican Science and Proctical Center of Cardiology, Minsk, Belarus

4 Research and Development, Siemens Healthcare Diagnostics GmbH, Austria

Key words heart, MR-imaging, cardiac function, myocardial relaxation time mapping, myocardial perfusion, pitfalls

received 08.04.2019

accepted 29.07.2019

Bibliography

DOI https://doi.org/10.1055/a-0999-5716

Published online: 20.11.2019

Fortschr Röntgenstr 2020; 192: 246-256

(c) Georg Thieme Verlag KG, Stuttgart · New York ISSN 1438-9029

Correspondence

Dr. Ursula Reiter

Radiology, Medizinische Universität Graz,

Auenbruggerplatz 9, 8036 Graz, Austria

Tel.: ++ 43/3 16/38583347

ursula.reiter@medunigraz.at

\section{ABSTRACT}

Background Cardiac magnetic resonance imaging (MRI) represents the established reference standard method for the assessment of cardiac function and non-invasive evaluation of myocardial tissue in a variety of clinical questions, wherein quantification of cardiac parameters gains growing diagnostic and differential-diagnostic importance. This review aims to summarize established and newly emerging quantitative parameters, which are assessed in routine cardiac MRI. Interrelations and interdependencies between metrics are explained, and common factors affecting quantitative results are discussed.

Method The review is based on a PubMed literature research using the search terms "cardiac magnetic resonance" and "quantification", "recommendations", "quantitative evaluation/assessment”, "reference method”, “reference/normal values", "pitfalls" or "artifacts" published between 20002019.

Results and Conclusion Quantitative functional, phase contrast, and perfusion imaging, as well as relaxation time mapping techniques give opportunity for assessment of a large number of quantitative cardiac MRI parameters in clinical routine. Application of these techniques allows for characterization of function, morphology and perfusion of the heart beyond visual analysis of images, either in primary evaluation and comparison to normal values or in patients' follow-up and treatment monitoring. However, with implementation of quantitative parameters in clinical routine, standardization is of particular importance as different acquisition and evaluation strategies and algorithms may substantially influence results, though not always immediately apparent.

\section{Key Points:}

- Clinical cardiac MRI provides numerous functional and morphological quantitative parameters.

- Quantitative cardiac MRI enables assessment of diffuse and global myocardial alterations.

- Standardized data acquisition/evaluation is the prerequisite for diagnostic use of quantitative cardiac MRI parameters.

\section{Citation Format}

- Reiter U, Reiter C, Kräuter C et al. Quantitative Clinical Cardiac Magnetic Resonance Imaging. Fortschr Röntgenstr 2020; 192: 246-256

\section{ZUSAMMENFASSUNG}

Hintergrund Die kardiale Magnetresonanztomografie (MRT) stellt sowohl in der Beurteilung der Herzfunktion als auch zur nichtinvasiven Gewebsanalyse des Myokards in vielen klinischen Fragestellungen die Referenz-Standard-Methode dar. Speziell die Quantifizierung kardialer Parameter nimmt eine immer zentralere diagnostische und differenzialdiagnostische Rolle ein. Im vorliegenden Review sollen etablierte und vielversprechende neue quantitative Herz-MRT-Parameter der klinischen Routine zusammengefasst, ihre Zusammenhänge beschrieben sowie ihre Abhängigkeiten von substanziellen Einflussfaktoren dargestellt werden.

Methode Die Übersichtsarbeit basiert auf einer PubMedLiteraturrecherche zu den Begriffen „cardiac magnetic resonance“ und „quantification“, „recommendations“, „quantitative evaluation/assessment“, „reference method“, „reference/ normal values“, „pitfalls“ sowie „artifacts“ innerhalb des Publikationszeitraums 2000-2019. 
Ergebnisse und Schlussfolgerung Funktionelle, Phasenkontrast- und Perfusionsbildgebung sowie Relaxationszeit-Kartierung ermöglichen die Erfassung einer Vielzahl quantitativer Herz-MRT-Parameter. Diese erlauben eine über die visuelle Beurteilung von Herz-MRT-Bildern hinausgehende Charakterisierung der Funktion, Morphologie und Perfusion des Herzens, sei es im Vergleich zu Normalwerten oder im Therapie- verlauf. Bei der Interpretation ausgewerteter Herz-MRTParameter in der klinischen Routine muss allerdings zunehmend auf Standardisierung geachtet werden, da Aufnahmetechniken und Auswertealgorithmen quantitative Ergebnisse maßgeblich - jedoch mitunter nicht unmittelbar erkennbar beeinflussen können.

\section{Introduction}

Currently, cardiac magnetic resonance imaging (MRI) represents the reference standard method in many clinical situations both for the evaluation of heart function as well as for non-invasive tissue analysis of the myocardium [1-3]. The quantification of cardiac and myocardial, functional and morphological parameters plays an increasingly central role in general cardiac diagnostics and differential diagnosis. While volumetric and blood flow-based cardiac MRI parameters can be used to define ventricular and atrial function, myocardial hypertrophy and dysfunction, as well as shunt and heart valve regurgitation volumes $[4,5]$, relaxation time mapping techniques allow quantitative morphological differentiation of global and regional myocardial changes, e. g. in the context of myocarditis, cardiac amyloidosis, Anderson-Fabry disease or cardiac iron storage disorders [6]. In addition, the quantification of myocardial perfusion, which could contribute to the diagnosis of multi-vascular and non-obstructive coronary vascular diseases, is becoming increasingly employed [7, 8].

This review aims to summarize established and new, quantitative routine cardiac MRI parameters as well as describe their significance and interconnections. A major challenge in the interpretation of quantitative cardiac MRI parameters lies in the definition of their normal values, which typically depend on both the scanning technique and evaluation algorithm [9]. Although the technical basics of cardiac MRI = Magnetic Resonance Imaging are only briefly discussed and reference is made to detailed reviews (among others [10-14]), substantial influencing factors on quantitative cardiac MRI parameters will be presented in more detail.

\section{Cine Imaging}

Cine imaging refers to the time-resolved representation of individual cardiac phases during a heartbeat. The k-space-segmented balanced steady state free precession (bSSFP) sequence is currently the standard technique for acquiring cine series. For patients with metallic cardiac implants (heart valves, pacemakers, implantable cardioverter defibrillators, etc.), the k-space segmented flash sequence (fast low-angle shot) is an alternative imaging technique with reduced artifact susceptibility $[13,15]$. Cine imaging during irregular heartbeat and/or respiration can be performed using real-time cine protocols. Using new compressed-sensing algorithms, temporal resolutions ( $\leq 45 \mathrm{~ms}$ [1]) and spatial resolutions comparable to k-space-segmented standard sequences can be achieved [16].

\section{Volumetric Function Parameters}

Cardiac MRI is considered the reference standard method for determining volumetric ventricular function parameters. In the clinical routine, standard evaluations include the left ventricular (and, depending on the clinical question, the right ventricular) end-diastolic (EDV) and end-systolic (ESV) volumes, stroke volume (SV), cardiac output (CO), ejection fraction (EF) and myocardial muscle mass [1, 2]. Volumetric quantities are given as absolute values or normalized to the body size. Due to their high reproducibility and accuracy, these are important parameters in cardiac diagnostics as well as for therapy and follow-up controls. It should be noted that volumetric cardiac MRI function parameters differ methodologically from echocardiographic, computed tomography or single-photon emission computed tomography (SPECT) function parameters limiting comparability of parameters throughout techniques [17-19].

Apart from the dependence of volumetric cardiac MRI function parameters on gender and age, both the acquisition technique (bSSFP or flash) and the evaluation strategy (manual, semi-automatic or automatic segmentation, inclusion or exclusion of papillary muscle, type of base level definition) must be taken into account when interpreting the data and comparing it with normal values ( $\triangleright$ Fig. 1). According to the guidelines for standardized cardiac MRI evaluation, it is recommended to segment myocardial trabeculae and papillary muscles to the myocardium to quantify muscle mass, but to assign them to the blood volume for the assessment of ejection fraction and ventricular stroke volume $[4,9]$.

With the implementation of robust automatic segmentation algorithms, all cardiac phases can be routinely evaluated volumetrically, and new functional parameters can be derived from the ventricular volume-time curve and its time derivative $(\mathrm{dV} / \mathrm{dt})$ ( Fig. 2); dV/dt curves represent ventricular blood flow rates in which the systolic minimum represents the peak ejection rate (PER), the diastolic maximums the early peak filling rate $\left(P F R_{E}\right)$ and the peak filling rate at atrial contraction $\left(P F R_{A}\right)$. The maximum filling rates, their ratios $\left(P F R_{E} / P F R_{A}\right)$, and filling rates normalized to the end-diastolic volume ( $\mathrm{PFR}_{\mathrm{E}} / \mathrm{EDV}, \mathrm{PFR}_{\mathrm{A}} / \mathrm{EDV}$ ) have the potential to characterize diastolic ventricular function and classify diastolic dysfunction without additional measurements [20, 21]. While ventricular function parameters are usually evaluated from cine short axis image stacks covering the ventricles, additional cine series in short or long axis orientation are required for volumetric evaluation of the left and right atrial function ( $>$ Fig. 2). Based on the biphasic atrial volume-time curve, maximum and minimum atrial volumes can be evaluated, and the total (TEF), passive (PEF) 

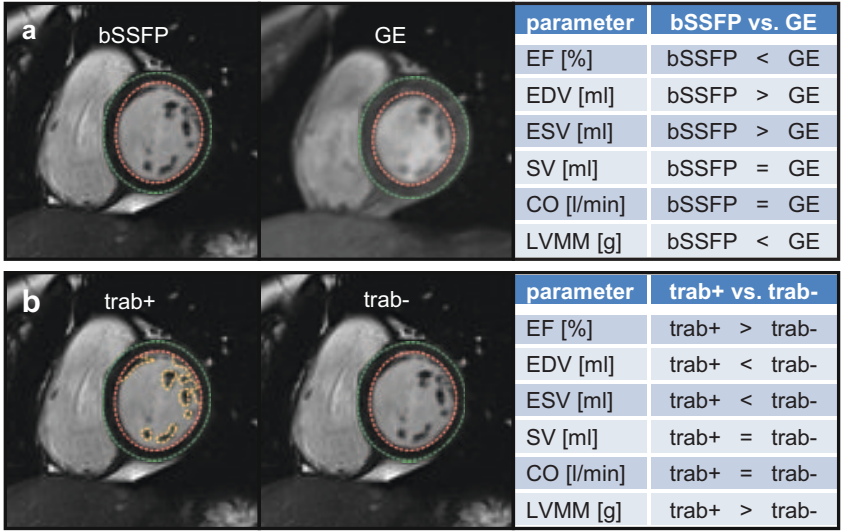

- Fig. 1 Impact of acquisition and evaluation on left ventricular volumetric function parameter and myocardial mass. a bSSFP compared to flash-acquisition. $\mathbf{b}$ Trabeculae and papillary muscles included to the myocardium (Trab+) compared to trabeculae and papillary muscles excluded from the myocardium (Trab-).

and contractile (CEF) atrial ejection fraction can be calculated [22]. Atrial function parameters can - analogous to echocardiography - also be estimated from biplanar area-length measurements, whereby atrial volumes and ejection fractions are overestimated compared to volumetric evaluation [9].

Irregular heartbeat poses a methodical limitation of all volumetric cardiac MRI function parameters. Although real-time imaging independent of respiration or heart rhythm allows the acquisition of cine series with virtually no movement artifacts, in the case of an irregular heartbeat, ventricular volumes differ generically from heartbeat to heartbeat. In studies including subjects with irregular heartbeat, evaluation of volumetric function parameters was performed by acquiring real-time cine series over several heartbeats and selecting comparable heartbeats for analysis [23, 24].

\section{Myocardial Function Parameters}

Together with the myocardial mass, myocardial wall thickness can be obtained from the segmentation of the myocardium for the diagnosis of myocardial hypertrophy and vitality, as well as systolic-to-diastolic wall thickness changes for the assessment of global and regional myocardial kinetics [4]. Regional parameters are typically reported according to the American Heart Association (AHA) 17-segment model. When evaluating the wall thickness, care must be taken that the papillary muscle and trabecula are not segmented to the myocardium and that the aortic outflow tract does not falsify the wall thickness of the basal anterior septal myocardial segment.

Cine tagging imaging is considered the reference standard method for the analysis of ventricular wall deformations (longitudinal, radial and circumferential strains) and deformation rates (strain rates) [25]. Feature tracking allows the evaluation of ventricular and atrial strains as well as strain rates based on routine cine series, allowing myocardial wall deformation to be detected without additional measurements in routine imaging ( $\triangleright$ Fig.3). Strains and strain rates vary, however, both regionally
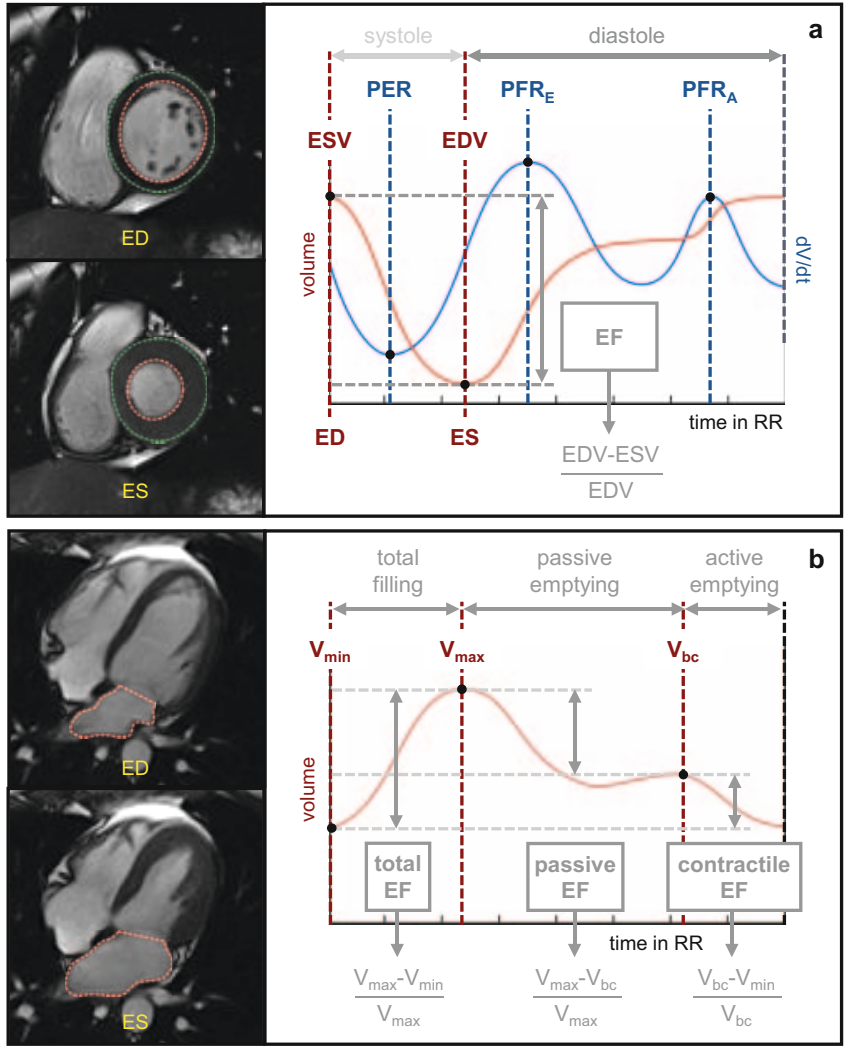

- Fig. 2 Schematic diagram of ventricular and atrial volume-time curves. a Ventricular volume-time curve (red graph) is shown together with its time derivative $\mathrm{dV} / \mathrm{dt}$ (blue graph), the maximal ventricular emptying (PER) and filling rates $\left(\mathrm{PFR}_{\mathrm{E}}, \mathrm{PFR}_{\mathrm{A}}\right)$. $\mathbf{b}$ Atrial volume-time curve for determination of the total, passive, and contractile atrial ejection fractions derived from maximal, minimal and before atrial contraction volumes. ED, ventricular end-diastole; $\mathrm{ES}$, ventricular end-systole.

(basal/midmyocardial/apical, endocardial/epicardial) and depending on the acquisition technique (feature tracking, displacement encoding with stimulated echoes (DENSE) imaging, cine tagging). In contrast to the volumetric function parameters, strains and strain rates differ depending on the specific evaluation software, which significantly limits the interpretation of myocardial strains in clinical routine and their comparison with published normal values [26, 27].

\section{Heart Valve Function}

In cine series, jets caused by heart valve stenoses or insufficiencies are visualized as signal cancellations; their appearance depends significantly on the parameters of the acquisition sequence (especially echo time) and the turbulent nature of the blood flow in the jet. Consequently, this phenomenon cannot be used to quantify cardiac valve stenoses or insufficiencies. However, planimetric evaluation of cine series acquired in the valve plane allow for determination of valvular opening area, and valve stenoses can be graded in accordance with echocardiography, the reference standard method $[28,29]$. 


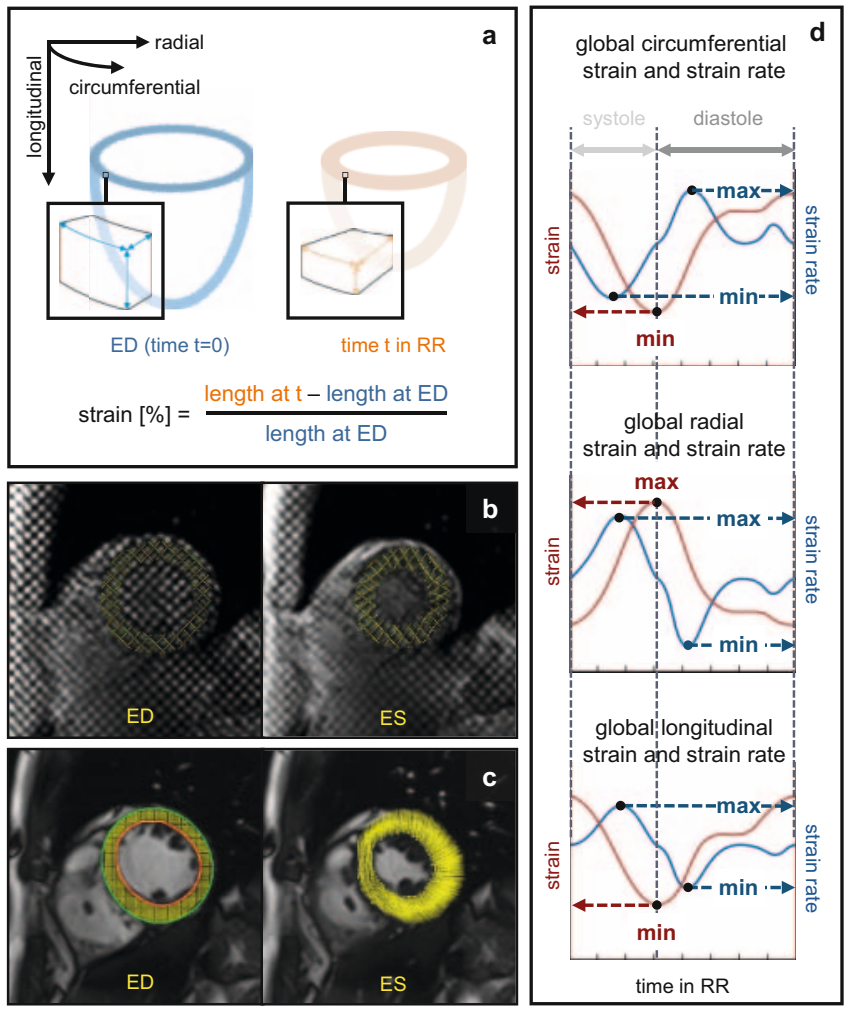

- Fig. 3 Ventricular strains and strain rates. a Schematic drawing illustrating the definition of circumferential, radial and longitudinal strains. $\mathbf{b}$ In tagging-analysis, myocardial strains are derived from tracing the deformation of an initially tagged grid (yellow lines). c In feature-tracking displacement of myocardial pixels during the heartbeat are modelled from standard cine series. $\mathbf{d}$ Representative time courses of global left ventricular strains (red line graphs) and their time derivatives (strain rates, blue line graphs).

\section{Phase-contrast Imaging}

The phase contrast technique allows the quantification of blood flow and myocardial velocities in any spatial direction [30, 31]. In cardiac MRI, the technique is typically used as a cine technique with unidirectional velocity coding perpendicular to the acquisition plane (through-plane) and called 2D flow, whereby the two dimensions refer to time resolution and unidirectional velocity measurement. Using acceleration techniques, 4D flow measurements, i. e. the recording of the temporally resolved tridirectional velocity field in a volume, become applicable for clinical routine [32].

2D flow measurements are typically acquired orthogonally to the assumed main flow direction through a selected cross-section (e. g. orthogonally to a vessel or parallel to atrio-ventricular heart valve planes). These can be used to determine temporal changes of the cross-sectional area, the maximum velocity, average speed and blood flow through the cross-section, as well as the corresponding temporally integral flow volume in the cardiac interval. Using multiplanar reconstruction, analogous quantities can be determined from 4D flow measurements for each crosssection within the recorded volume ( $\triangleright$ Fig. 4 ). Both methods offer different advantages and disadvantages. Advantages
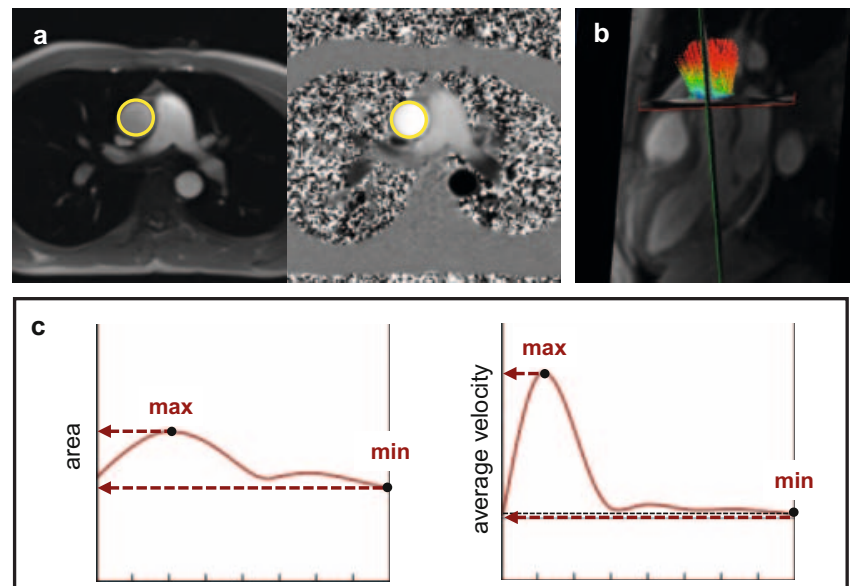

flow $=$ area $\times$ average through-plane velocity
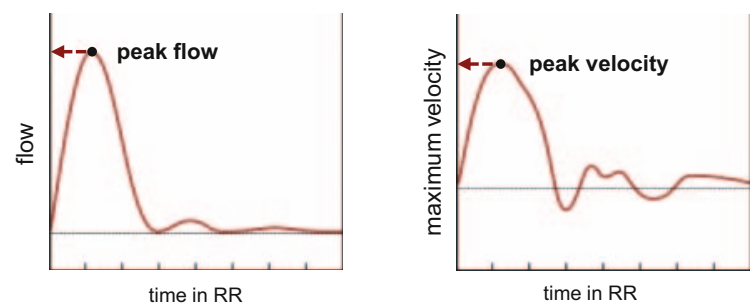

- Fig. 4 2D and 4D phase contrast imaging derived parameters in the aorta ascendens. a Segmentation of the aortic vessel crosssection (yellow line) in a 2D-flow measurement with the imaging plane orientated orthogonal to the vessel course. b Multiplanar reconstruction of an evaluation plane (red plane) in a 4D flow measurement covering the entire aorta ascendens for a posteriori evaluation of aortic blood flow. Visualization of blood velocity field through the aorta (vectors) enables optimized alignment of the evaluation plane orthogonal to the direction of the blood flow. c Representative time courses of the vessel cross-section area, average and maximum through-plane velocity through the segmented vessel cross-section, and the calculated blood flow through the vessel cross-section.

of 2D over 4D flow measurement include short scan times (so that data can be acquired under breath holding) and the possibility of optimize velocity coding (VENC, as small as possible to maximize velocity-to-noise ratio, but greater than maximum velocities to avoid aliasing and thus potential errors in evaluation $[30,31,33])$. Compared to 2D flow measurements, 4D flow measurements are characterized by simple planning and a-posteriori analysis of any measurement plane of interest from a data set [32].

\section{Quantification of Blood Flow Volumes}

The central application of the phase contrast method in routine cardiac MRI is the quantification of aortic $\left(Q_{A}\right)$ and pulmonary blood flow volumes $\left(Q_{P}\right)$ in the heartbeat. In the absence of cardiac/cardiovascular shunts and/or heart valve insufficiencies, $\mathrm{Q}_{\mathrm{A}}$ and $\mathrm{Q}_{\mathrm{P}}$ correspond to the left and right ventricular stroke volume [5, 34]. By comparing the blood flow volumes with each other $\left(Q_{P} / Q_{A}\right.$ ratio) and with the volumetric ventricular stroke volumes, both shunt volumes and regurgitation volumes of the 
atrio-ventricular valves can be estimated ( $\triangleright$ Fig. 5). Regurgitation volumes of semilunar valves can be determined directly from the time course of aortic and pulmonary phase contrast measurements. While no effective blood flow through the vessel crosssection can be detected after end-systolic valve closure, valve insufficiencies cause a diastolic reflux volume ( $\mathbf{F i g}$. 6 ).

The comparative interpretation of volumetric and phase-contrast-based blood flow volumes is decisively determined by the accuracy of the volumetric evaluation, accuracy of the phase contrast measurement and physiological variations between the measurements. Volumetric ventricular stroke volumes can be verified using the (diastolic) inflow volume measured through the atrio-ventricular valves [35]. The accuracy of the results of phase contrast measurements is limited by the segmentation, the choice of measuring plane, as well as possible spatially variable background phases (exact orthogonality to the vessel plays a secondary role, since the resulting underestimation of the velocity is compensated by the overestimation of the cross-sectional area). Background phases can be minimized by measuring near the isocenter and by adequate correction in post-processing [30, 31]. 4D flow measurements have the potential to further improve the accuracy of phase contrast measurements, since physiological variations between individual 2D phase contrast measurements are eliminated and (valve) planes can be adapted accordingly over the course of time [32].

\section{Maximum and Peak Velocities}

Measurement of maximum and peak velocities plays an important role in the determination of the severity of valve stenoses as well as in the evaluation of diastolic heart function.

Peak velocities in jets caused by valve stenoses can be determined in accordance with the reference standard method, echocardiography. The prerequisite for this, however, is that in the phase contrast measurement short echo times and adequate temporal and spatial resolution are selected, the 2D phase contrast plane in the vena contracta is aligned orthogonally to the velocity, and velocities are evaluated without averaging from individual pixels [4, 30, 31, 36].

Analogous to echocardiography, the early (E) and late diastolic (A) peak blood flow profiles over the atrio-ventricular valves can be used to determine the transmitral or transtricuspidal E/A ratio as a parameter of ventricular diastolic function ( $\triangleright$ Fig. 7). Other phase contrast parameters of the diastolic function are [37-39]: the systolic (S) and early diastolic (D) pulmonary vein peak velocity, as well as the S/D ratio for grading diastolic dysfunction, myocardial early diastolic velocity (e'), and E/e' ratio for estimating left ventricular filling pressure. Although studies show a good correlation of these parameters to echocardiography [39], diastolic function parameters are routinely not evaluated due to the necessity of additional measurements and the lack of standardization of acquisition protocols. It should be noted that phase contrast protocols with higher temporal resolution $(15-20 \mathrm{~ms}[37,39])$ and adapted VENCs $(100-180 \mathrm{~cm} / \mathrm{s}$ for transmitral, $10-30 \mathrm{~cm} / \mathrm{s}$ for myocardial measurements [37]) have been used in studies to evaluate diastolic functional parameters. With the increasing implementation of 4D flow measurements, evaluation of diastolic func-
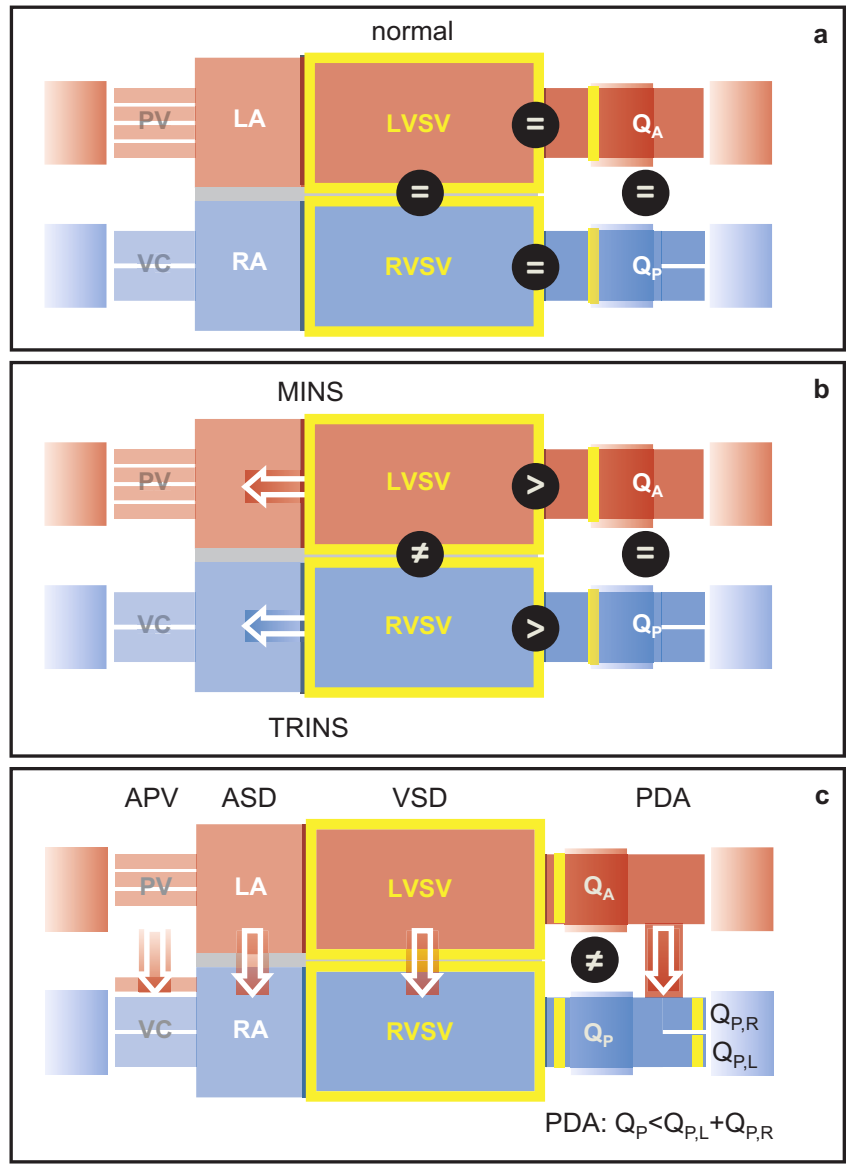

\begin{tabular}{|c|c|c|c|}
\hline $\begin{array}{l}\text { single } \\
\text { condition }\end{array}$ & $\begin{array}{l}\text { volumetry vs. } \\
\text { aortic/pulmo flow }\end{array}$ & $\begin{array}{l}\text { aortic vs. } \\
\text { pulmo flow }\end{array}$ & typical pitfalls \\
\hline normal & $\begin{aligned} \text { LVSV } & =Q_{A} \\
\text { RVSV } & =Q_{P}\end{aligned}$ & $Q_{A}=Q_{P}$ & \multirow{8}{*}{$\begin{array}{l}\text { LVSV } \neq \text { RVSV: } \\
\text { - ED/ES baseplane } \\
\text { definition incorrect } \\
\text { - LV/RV segmentation of } \\
\text { trabecle inconsitant } \\
\text { - RV not fully covered (by } \\
\text { short-axis stack) } \\
Q_{A} \neq Q_{\mathrm{p}} \text { : } \\
\text { - different background } \\
\text { phases in measurements } \\
\text { SV } \neq Q_{\mathrm{A}} \neq \mathrm{Q}_{\mathrm{p}} \text { : } \\
\text { - Different breath hold state } \\
\text { during measurements } \\
\text { - Different heart rates } \\
\text { during single } \\
\text { measurements } \\
\text { - large time delay between } \\
\text { single measurements }\end{array}$} \\
\hline MINS & LVSV $>Q_{A}$ & $Q_{A}=Q_{P}$ & \\
\hline TRINS & $R V S V>Q_{p}$ & $Q_{A}=Q_{P}$ & \\
\hline AINS & $\begin{aligned} \text { LVSV } & =Q_{A} \\
\text { RVSV } & =Q_{P}\end{aligned}$ & $Q_{A}=Q_{P}$ & \\
\hline PINS & $\begin{aligned} \text { LVSV } & =Q_{A} \\
\text { RVSV } & =Q_{P}\end{aligned}$ & $Q_{A}=Q_{P}$ & \\
\hline $\begin{array}{l}\text { APV or } \\
\text { ASD }\end{array}$ & $\begin{aligned} \text { LVSV } & <\mathrm{RVSV} \\
\text { LVSV } & =\mathrm{Q}_{\mathrm{A}} \\
\mathrm{RVSV} & =\mathrm{Q}_{\mathrm{P}}\end{aligned}$ & $Q_{A}<Q_{P}$ & \\
\hline VSD & $\begin{array}{l}\text { LVSV }=\text { RVSV } \\
\text { LVSV }>Q_{A} \\
\text { RVSV }=Q_{P}\end{array}$ & $\begin{array}{l}Q_{A}<Q_{P} \\
Q_{P}=Q_{l i P}+Q_{\text {reP }}\end{array}$ & \\
\hline PDA & $\begin{aligned} \text { LVSV } & >\text { RVSV } \\
\text { LVSV } & =Q_{A} \\
\text { RVSV } & =Q_{P}\end{aligned}$ & $\begin{array}{l}Q_{A}>Q_{P} \\
Q_{P}<Q_{\text {liP }}+Q_{\text {reP }} \\
Q_{A}=Q_{\text {liP }}+Q_{\text {reP }}\end{array}$ & \\
\hline
\end{tabular}

- Fig. 5 Interpretation schemes for combined analysis of volumetric ventricular stroke volumes and phase contrast imaging derived blood flow volumes $\mathbf{a}$ in absence of atrio-ventricular valve insufficiencies and absence of cardiac/cardiovascular shunts, $\mathbf{b}$ in presence of atrioventricular valve insufficiencies and absence of cardiac/cardiovascular shunts and, $\mathbf{c}$ in presence of cardiac/cardiovascular shunts. d Summary on relations between volumetric ventricular stroke volumes and phase contrast imaging derived blood flow volumes. LA/RA, left/right atrium; PV, pulmonary vein; VC, venae cavae; MINS/ TRINS, mitral/tricuspidal insuffiency; PVF, anomalous pulmonary venous connection; ASD, atrial septal defect; VSD, ventricular septal defect; PDA, patent ductus arteriosus; LVSV/RVSV, left/right ventricular stroke volume; $Q_{A}$, aortic flow volume per heartbeat; $Q_{p}$, pulmonary flow volume per heartbeat, $Q_{P, R} / Q_{P, L}$, flow volume through the right/left pulmonary artery branch. 

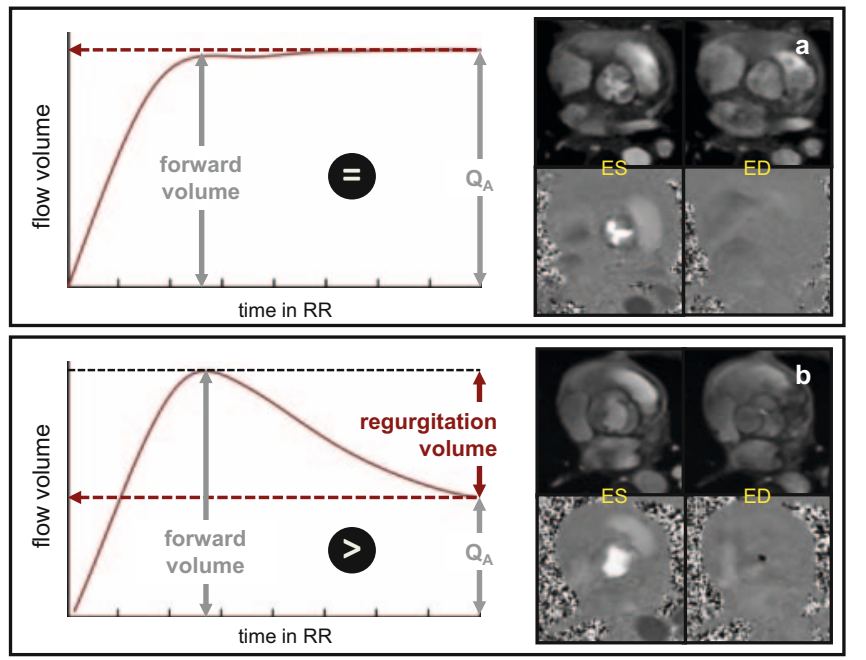

- Fig. 6 Evaluation of the regurgitation volume in aortic valve insufficiency. a Representative time course of the blood volume through the aortic vessel cross-section in absence of an aortic valve insufficiency. The forward blood volume after systolic aortic valve closure equals the aortic flow volume within the cardiac cycle $Q_{A}$. b Representative time course of the blood volume through the aortic vessel cross-section in presence of an aortic valve insufficiency. After systolic aortic valve closure backward flow is observed through the aortic vessel cross-section. The regurgitation volume is calculated as the difference between forward volume and QA.

tion without additional measurements could become established in clinical routine [32].

\section{Perfusion Imaging}

In cardiac MR perfusion imaging, the influx of intravenous gadolinium-based contrast agent into the myocardium is analyzed under pharmacological stress and/or resting conditions using single-shot gradient echo (GRE) sequences (flash, bSSFP, and GRE-EPI hybrid sequences, the former due to the lowest artifact susceptibility most commonly used [40]). Perfusion series are typically recorded in 3 short-axis and one long-axis plane in order to cover as many of the $17 \mathrm{AHA}$ segments as possible. In clinical routine, the interpretation of regionally delayed arrival of contrast agent in perfusion series is primarily performed qualitatively. Stress and resting perfusion series are analyzed in order to differentiate between myocardial ischemia and a dark rim artifact mimicking a subendocardial perfusion defect [41, 42].

In addition to the potential for objective detection of regional perfusion deficits, quantitative perfusion measurements offer the possibility to detect global or diffuse myocardial perfusion changes, such as in non-obstructive coronary heart disease [7, 8, 43]. At present, the myocardial perfusion reserve index (MPRI) is the most established cardiac MRI perfusion parameter. The MPRI is the ratio of the upslopes of the myocardial signal intensity curves during first pass of contrast agent under stress and rest $(\triangleright$ Fig. 8). The rationale of this semi-quantitative parameter is that upslopes are a measure of myocardial perfusion so that their quotient can be interpreted as a non-invasive correlate to the
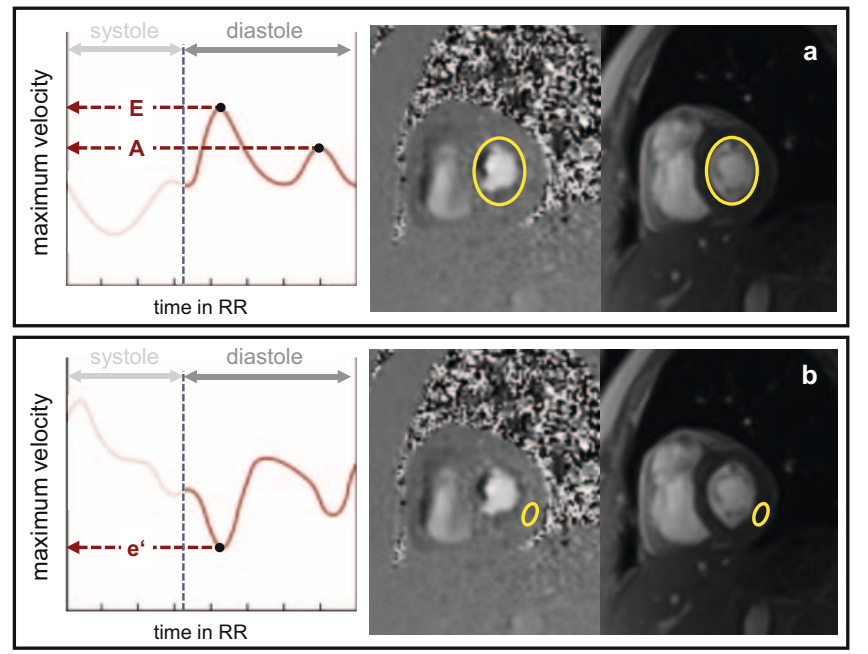

- Fig.7 Evaluation of diastolic left ventricular function parameters from transmitral 2D-flow measurements. a Representative time course of the transmitral maximum velocity (red graph) with early (E) and late (A) diastolic peak velocities. b Representative time course of myocardial tissue maximum velocity (red graph) with the early diastolic peak velocity e'.

coronary fractional flow reserve (FFR) [44-46]. A key limitation of the index, despite its conceptual simplicity, is the dependence on the protocol parameters (e.g., amount of contrast agent, sequence parameters, time between stress and resting perfusion acquisition), and the evaluation software used [47].

A more universal absolute quantification of myocardial perfusion requires the conversion of signal intensity curves in the myocardium and left ventricular blood pool into corresponding contrast agent concentration curves [8]. This results, however, in special requirements for data acquisition and/or contrast agent application. Although the feasibility of even pixel-by-pixel perfusion quantification has been demonstrated [44], this method is not yet clinically established [48].

\section{Relaxation Time Mapping}

Myocardial relaxation time mapping refers to the pixel-by-pixel estimation of magnetic relaxation times ( $\left.\mathrm{T} 1, \mathrm{~T} 2, \mathrm{~T} 2^{*}\right)$ of the myocardium. Analogously to T1-, T2- or T2*-weighted images, relaxation time maps display regional myocardial changes as regionally different relaxation times. Their potential lies primarily in the fact that by segmentation of the myocardium or myocardial evaluation regions, mean myocardial relaxation times can be determined and compared with normal values $[6,9]$. Thus, not only regional but also diffuse and global myocardial changes can be identified ( $\triangleright$ Fig. 9).

Cardiac relaxation time maps are calculated from image series with a varying sequence parameter using known relationships between relaxation times and signal intensities. Current mapping methods allow the image series to be captured within one breath hold interval. For T1 mapping, the Modified Look-Locker Inversion recovery (MOLLI) sequence is the most established method, where $\mathrm{T} 1$ values are obtained from single-shot bSSFP image series 


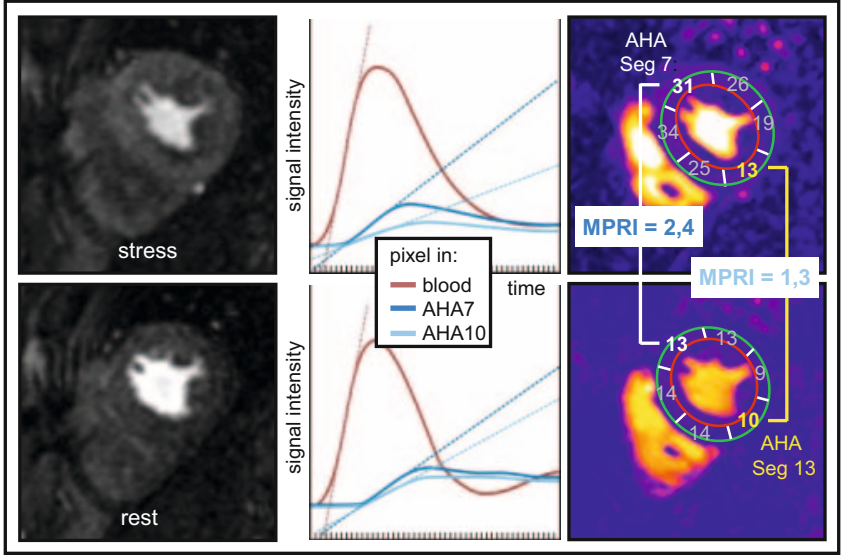

- Fig. 8 Assessment of the myocardial perfusion reserve index (MPRI). Motion corrected perfusion images (left) can be employed to derive pixel-wise signal intensity time couses during first pass of contrast agent (middle). Pixel-wise upslope maps during first pass of contrast agent can be evaluated regionally or in AHA-segments, respectively (right). MPRI is defined as stress-to-rest upslope ratio, which is reduced in the ischemic AHA segment 10 compared to the non-ischemic AHA segment 7. with varying inversion time [49-51]. To estimate myocardial T2 times, for example, single-shot GRE image series with varying T2 preparation times are used $[51,52]$ and $T 2 *$ times are finally calculated from segmented multiecho GRE images with different echo times $[6,52]$.

\section{T1 and T2 Mapping}

While in native myocardial T1 maps, myocardial fibrosis and myocardial edema are associated with elevated T1 values, in T1 maps after contrast agent application (post-contrast T1 maps), areas with increased extracellular space in myocardial fibrosis or necrosis are associated with decreased T1 values corresponding to the higher contrast agent distribution volume [49, 53]. Post-contrast $\mathrm{T} 1$ values are determined not only by the morphological change of the myocardium but also by the amount of contrast agent applied, the time after application of the contrast agent and the contrast agent wash out behavior of the myocardium. It is therefore common to calculate the extracellular volume fraction (ECV), which is largely independent of the contrast agent kinetics $[50,54]$ and reflects the relative distribution volume of the contrast agent in the myocardium ( $\triangleright$ Fig. 9). Since myocardial edema significantly limits the association between elevated native

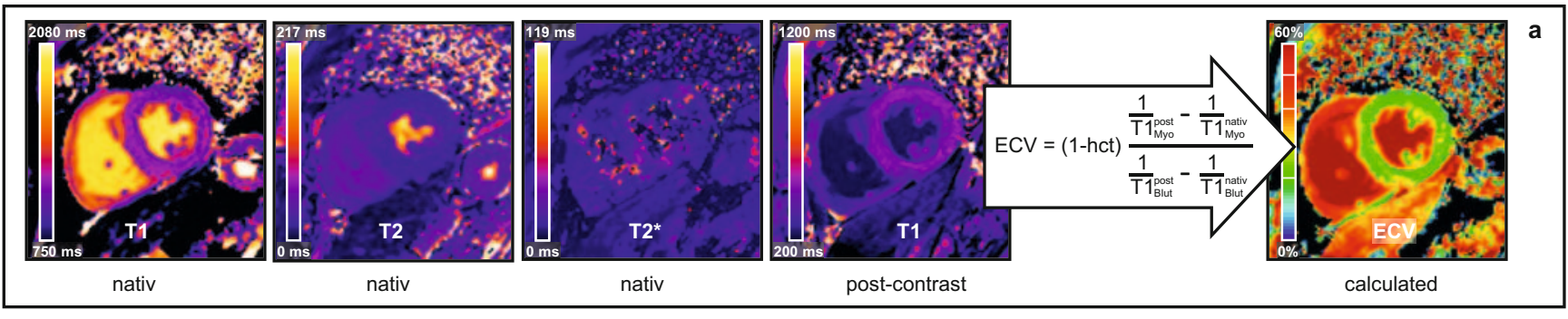

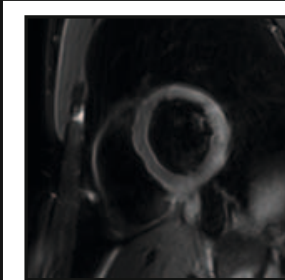

T2-weighted

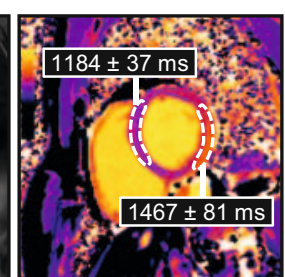

$\mathrm{T} 1$

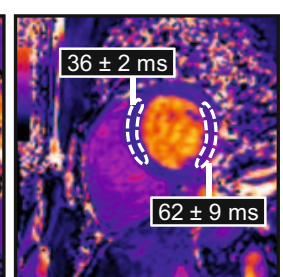

T2

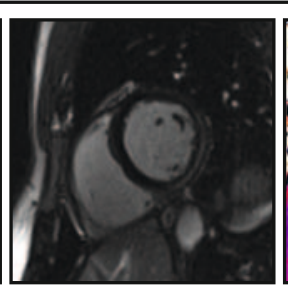

late enhancement

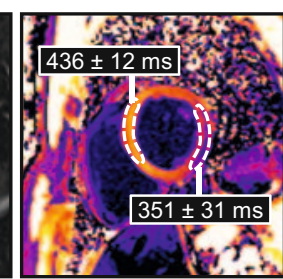

T1-post

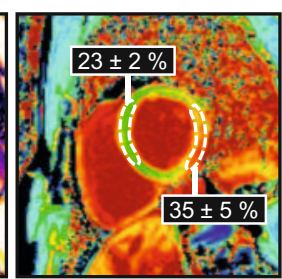

$\mathrm{ECV}$ with hct $=0,39$

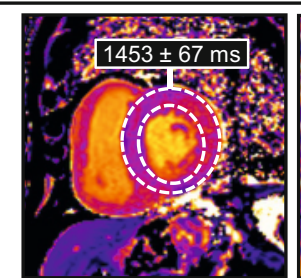

$\mathrm{T} 1$

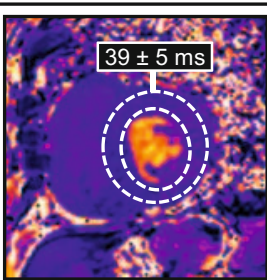

T2

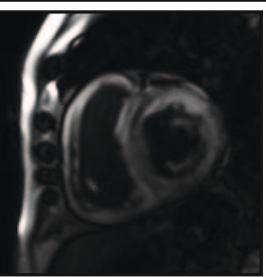

late enhancement (magnitude und phase sensitive)

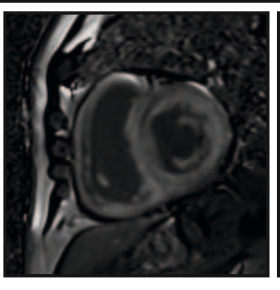$$
\text { e) }
$$

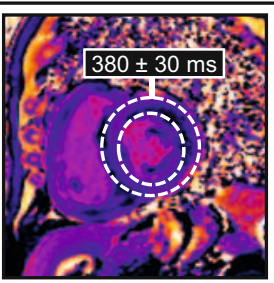

T1-post

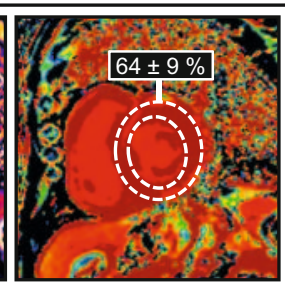

ECV with hct $=0,48$

- Fig. 9 Myocardial relaxation time maps. a Native T1-, T2, and T2* maps together with a post-contrast T1 map (T1-post). b Regional T1, T2, and ECV alterations in acute myocarditis. T1, T2, and ECV values in involved lateral myocardium, as visualized also in the T2 weighted and late enhancement images, are higher than in the non-involved septal myocardium. c Global T1 and ECV alterations in cardiac amyloidosis. Globally severely elevated native T1 in absence of myocardial edema (normal myocardial T2) has been introduced as superior diagnostic marker for cardiac amyloidosis, especially in case of atypical late enhancement. 


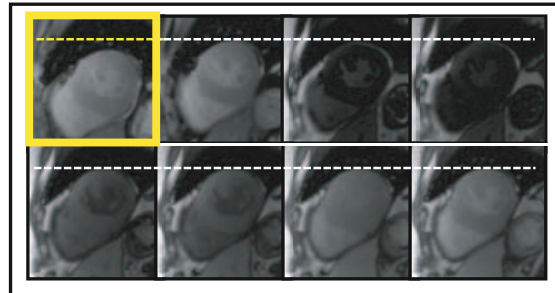

image series after motion correction

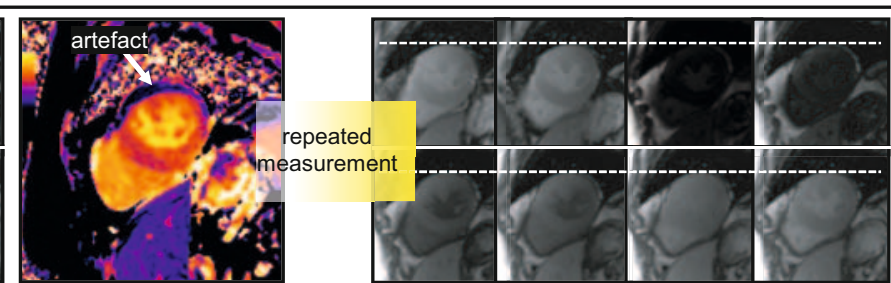

T1

image series after motion correction

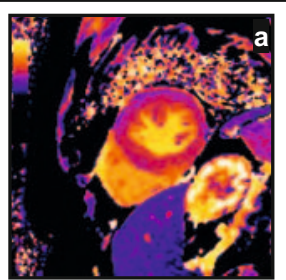

$\mathrm{T} 1$

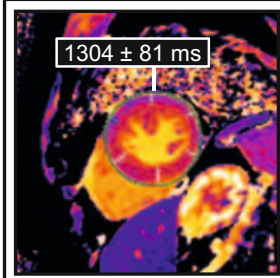

$0 \%$ offset

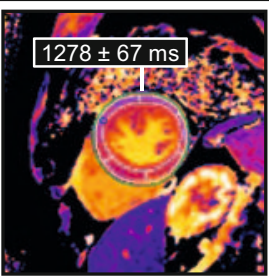

$20 \%$ offset

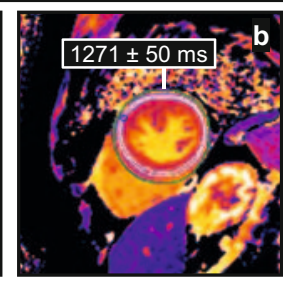

$40 \%$ offset

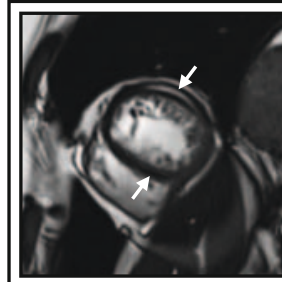

bSSFP

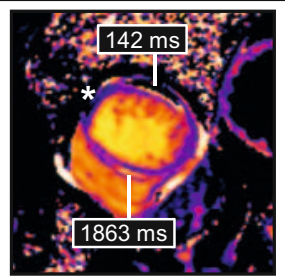

$\mathrm{T} 1$

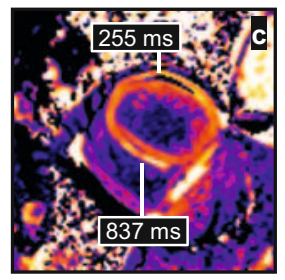

T1-post

- Fig. 10 Potential pitfalls in the interpretation and evaluation of relaxation time maps. a Motion between images. Motion correction algorithms typically employed prior to map calculations might fail to perfectly superpose pixel throughout image series (left). Resulting motion artefacts might be difficult to be identified in maps but can be recognized in motion corrected image series (yellow frame). b Partial volume of blood, fat, or pericardial fluid might impair average myocardial relaxation times in regions-of-interests at myocardial borders [57]. For comparison of relaxation times with normal ranges, standardized evaluation of maps is essential. c Pixels containing fat and myocardium might present with artificial T1 values in T1 maps [58]. As illustrated in the case of lipomatous metaplasia (arrows) T1 maps show low values in pixels predominantly containing fat (lateral), but demonstrate artificially high values in the septum and the lateral border zones. ${ }^{*}$ indicates motion artefact as described in a (compare T1-post map without motion artefact).

T1 and ECV values with myocardial fibrosis, it is recommended to interpret T1 and ECV maps together with additional T2 maps in which myocardial edema present with elevated T2 time [55]. While myocardial T1 and T2 time changes are not specific, they have been shown to increase cardiac MRI-based diagnostic accuracy in myocarditis, amyloidosis, Anderson-Fabry disease and cardiac iron storage disorders [6, 56].

Although the acquisition and evaluation of myocardial relaxation time maps is simple, the acquisition and evaluation technique must be taken into account in the interpretation of the values ( $\triangleright$ Fig. 10). Relaxation times determined pixel-by-pixel depend significantly on selected sequence and protocol parameters; regional relaxation time averages can be falsified by partial volume effects of the structures adjacent to the myocardium (blood, fat, pericardial fluid) $[57,58]$. Accordingly, normal values identical to clinical routine mapping protocols must be obtained, whereby acquisition and evaluation must be performed in a standardized manner $[6,50]$. If the pixel-by-pixel overlay of the image series is limited by (movement) artifacts, affected myocardial segments must be detected and excluded from the evaluation.

\section{T2* Mapping}

Reduced septal myocardial T2* times at $1.5 \mathrm{~T}$ are the reference standard method for non-invasive diagnosis and grading of cardiac hemochromatosis [6, 49]. For the severity classification of myocardial iron storage by $\mathrm{T} 2 *$ mapping, $1.5 \mathrm{~T} \mathrm{T2*}$ times of the basal septum over $20 \mathrm{~ms}$ are interpreted as normal, values less than $10 \mathrm{~ms}$ indicate severe myocardial iron storage [6, 60, 61]. The artifact susceptibility of the method and the lack of reference values for $3 \mathrm{~T}$ could contribute to establishing the reduction of myocardial T1 and T2 values caused by cardiac iron storage diseases as new diagnostic markers [6].

\section{Summary}

Quantitative functional, phase contrast, and perfusion imaging, as well as relaxation time mapping techniques give opportunity for assessment of a large number of quantitative cardiac MRI parameters in clinical routine.

Application of these techniques allows for characterization of function, morphology and perfusion of the heart beyond visual analysis of images, either in primary evaluation and comparison to normal values or in patients' follow-up and treatment monitoring. However, with implementation of quantitative parameters in clinical routine, standardization is of particular importance as different acquisition and evaluation strategies and algorithms may substantially influence results, though not always immediately apparent.

\section{Conflict of Interest}

Gert Reiter is employee of Research \& Developement, Siemens Healthcare Diagnostics $\mathrm{GmbH}$.

\section{Literatur}

[1] Kramer CM, Barkhausen J, Flamm SD et al. Society for Cardiovascular Magnetic Resonance Board of Trustees Task Force on Standardized Protocols. Standardized cardiovascular magnetic resonance (CMR) protocols 2013 update. J Cardiovasc Magn Reson 2013; 15: 91. doi:10.1186/1532429X-15-91 
[2] Puntmann VO, Valbuena S, Hinojar R et al. Society for Cardiovascular Magnetic Resonance (SCMR) expert consensus for CMR imaging endpoints in clinical research: part I - analytical validation and clinical qualification. J Cardiovasc Magn Reson 2018; 20: 67. doi:10.1186/s12968018-0484-5

[3] Peterzan MA, Rider OJ, Anderson LJ. The Role of Cardiovascular Magnetic Resonance Imaging in Heart Failure. Card Fail Rev 2016; 2: 115-122. doi:10.15420/cfr.2016.2.2.115

[4] Schulz-Menger ], Bluemke DA, Bremerich J et al. Standardized image interpretation and post processing in cardiovascular magnetic resonance: Society for Cardiovascular Magnetic Resonance (SCMR) board of trustees task force on standardized post processing. J Cardiovasc Magn Reson 2013; 15: 35,429X-15-35. doi:10.1186/1532-429X-15-35

[5] Fratz S, Chung T, Greil GF et al. Guidelines and protocols for cardiovascular magnetic resonance in children and adults with congenital heart disease: SCMR expert consensus group on congenital heart disease. J Cardiovasc Magn Reson 2013; 15: 51,429X-15-51. doi:10.1186/1532429X-15-51

[6] Messroghli DR, Moon JC, Ferreira VM et al. Clinical recommendations for cardiovascular magnetic resonance mapping of $\mathrm{T} 1, \mathrm{~T} 2, \mathrm{~T} 2{ }^{*}$ and extracellular volume: A consensus statement by the Society for Cardiovascular Magnetic Resonance (SCMR) endorsed by the European Association for Cardiovascular Imaging (EACVI). J Cardiovasc Magn Reson 2017; 19 : 75,017-0389-8. doi:10.1186/s12968-017-0408-9

[7] Merz CN, Pepine C], Walsh MN et al. Ischemia and No Obstructive Coronary Artery Disease (INOCA): Developing Evidence-based Therapies and Research Agenda for the Next Decade. Circulation 2017; 135: 10751092. doi:10.1161/CIRCULATIONAHA.116.024534

[8] Robinson AA, Salerno M, Kramer CM. Contemporary Issues in Quantitative Myocardial Perfusion CMR Imaging. Current Cardiovascular Imaging Reports 2019; 12: 9. doi:10.1007/s12410-019-9484-6

[9] Kawel-Boehm N, Maceira A, Valsangiacomo-Buechel ER et al. Normal values for cardiovascular magnetic resonance in adults and children. J Cardiovasc Magn Reson 2015; 17: 29,015-0111-7. doi:10.1186/ s12968-015-0111-7

[10] Ridgway JP. Cardiovascular magnetic resonance physics for clinicians: part I. J Cardiovasc Magn Reson 2010; 12: 71. doi:10.1186/1532-429X$12-71$

[11] Biglands JD, Radjenovic A, Ridgway JP. Cardiovascular magnetic resonance physics for clinicians: Part II. J Cardiovasc Magn Reson 2012; 14 : 66. doi:10.1186/1532-429X-14-66

[12] Krishnamurthy R, Cheong B, Muthupillai R. Tools for cardiovascular magnetic resonance imaging. Cardiovasc Diagn Ther 2014; 4: 104-125. doi:10.3978/j.issn.2223-3652.2014.03.06

[13] Ferreira PF, Gatehouse PD, Mohiaddin RH et al. Cardiovascular magnetic resonance artefacts. J Cardiovasc Magn Reson 2013; 15: 41. doi:10.1186/1532-429X-15-41

[14] Finn JP, Nael K, Deshpande V et al. Cardiac MR imaging: state of the technology. Radiology 2006; 241: 338-354

[15] Olivieri LJ, Cross RR, O'Brien KE et al. Optimized protocols for cardiac magnetic resonance imaging in patients with thoracic metallic implants. Pediatr Radiol 2015; 45: 1455-1464. doi:10.1007/s00247-015-3366-0

[16] Kido T, Kido T, Nakamura $M$ et al. Compressed sensing real-time cine cardiovascular magnetic resonance: accurate assessment of left ventricular function in a single-breath-hold. J Cardiovasc Magn Reson 2016; 18: 50,016-0271-0. doi:10.1186/s12968-016-0271-0

[17] Wood PW, Choy JB, Nanda NC et al. Left ventricular ejection fraction and volumes: it depends on the imaging method. Echocardiography 2014; 31: 87-100. doi:10.1111/echo.12331

[18] Pellikka PA, She L, Holly TA et al. Variability in Ejection Fraction Measured By Echocardiography, Gated Single-Photon Emission Computed Tomography, and Cardiac Magnetic Resonance in Patients With Coronary
Artery Disease and Left Ventricular Dysfunction. JAMA Netw Open 2018; 1: e181456. doi:10.1001/jamanetworkopen.2018.1456

[19] Reiter G, Reiter U, Rienmüller R et al. On the value of geometry-based models for left ventricular volumetry in magnetic resonance imaging and electron beam tomography: a Bland-Altman analysis. Eur J Radiol 2004; 52: 110-118

[20] Kawaji K, Codella NC, Prince MR et al. Automated segmentation of routine clinical cardiac magnetic resonance imaging for assessment of left ventricular diastolic dysfunction. Circ Cardiovasc Imaging 2009; 2: 476484. doi:10.1161/CIRCIMAGING.109.879304

[21] Nacif MS, Almeida ALC, Young AA et al. Three-Dimensional Volumetric Assessment of Diastolic Function by Cardiac Magnetic Resonance Imaging: The Multi-Ethnic Study of Atherosclerosis (MESA). Arq Bras Cardiol 2017; 108: 552-563. doi:10.5935/abc.20170063

[22] Kowallick JT, Morton G, Lamata P et al. Quantification of atrial dynamics using cardiovascular magnetic resonance: inter-study reproducibility. J Cardiovasc Magn Reson 2015; 17: 36,015-0140-2. doi:10.1186/ s12968-015-0140-2

[23] Contijoch F, Witschey WR, Rogers K et al. User-initialized active contour segmentation and golden-angle real-time cardiovascular magnetic resonance enable accurate assessment of LV function in patients with sinus rhythm and arrhythmias. J Cardiovasc Magn Reson 2015; 17: 37. doi:10.1186/s12968-015-0146-9

[24] Contijoch F, Rogers K, Rears H et al. Quantification of Left Ventricular Function With Premature Ventricular Complexes Reveals Variable Hemodynamics. Circ Arrhythm Electrophysiol 2016; 9: e003520. doi:10.1161/CIRCEP.115.003520

[25] Ibrahim el-SH. Myocardial tagging by cardiovascular magnetic resonance: evolution of techniques - pulse sequences, analysis algorithms, and applications. J Cardiovasc Magn Reson 2011; 13: 36,429X-13-36. doi:10.1186/1532-429X-13-36

[26] Augustine D, Lewandowski AJ, Lazdam M et al. Global and regional left ventricular myocardial deformation measures by magnetic resonance feature tracking in healthy volunteers: comparison with tagging and relevance of gender. J Cardiovasc Magn Reson 2013; 15: 8,429X-15-8. doi:10.1186/1532-429X-15-8

[27] Almutairi HM, Boubertakh R, Miquel ME et al. Myocardial deformation assessment using cardiovascular magnetic resonance-feature tracking technique. Br J Radiol 2017; 90: 20170072. doi:10.1259/bjr.20170072

[28] Kupfahl C, Honold M, Meinhardt G et al. Evaluation of aortic stenosis by cardiovascular magnetic resonance imaging: comparison with established routine clinical techniques. Heart 2004; 90: 893-901. doi:10.1136/hrt.2003.022376

[29] Schlosser T, Malyar N, Jochims M et al. Quantification of aortic valve stenosis in MRI-comparison of steady-state free precession and fast low-angle shot sequences. Eur Radiol 2007; 17: 1284-1290. doi:10.1007/s00330-006-0437-5

[30] Gatehouse PD, Keegan ], Crowe LA et al. Applications of phase-contrast flow and velocity imaging in cardiovascular MRI. Eur Radiol 2005; 15 : 2172-2184. doi:10.1007/s00330-005-2829-3

[31] Nayak KS, Nielsen JF, Bernstein MA et al. Cardiovascular magnetic resonance phase contrast imaging. J Cardiovasc Magn Reson 2015; 17 : 71,015-0172-7. doi:10.1186/s12968-015-0172-7

[32] Dyverfeldt P, Bissell M, Barker AJ et al. 4D flow cardiovascular magnetic resonance consensus statement. J Cardiovasc Magn Reson 2015; 17 : 72,015-0174-5. doi:10.1186/s12968-015-0174-5

[33] Gatehouse PD, Rolf MP, Graves MJ et al. Flow measurement by cardiovascular magnetic resonance: a multi-centre multi-vendor study of background phase offset errors that can compromise the accuracy of derived regurgitant or shunt flow measurements. J Cardiovasc Magn Reson 2010; 12: 5,429X-12-5. doi:10.1186/1532-429X-12-5

[34] Aquaro GD, Barison A, Todiere G et al. Cardiac magnetic resonance "virtual catheterization" for the quantification of valvular regurgitations 
and cardiac shunt. J Cardiovasc Med (Hagerstown) 2015; 16: 663-670. doi:10.2459/JCM.0000000000000245

[35] Krieger EV, Lee ], Branch KR et al. Quantitation of mitral regurgitation with cardiac magnetic resonance imaging: a systematic review. Heart 2016; 102: 1864-1870. doi:10.1136/heartjnl-2015-309054

[36] Defrance C, Bollache E, Kachenoura N et al. Evaluation of aortic valve stenosis using cardiovascular magnetic resonance: comparison of an original semiautomated analysis of phase-contrast cardiovascular magnetic resonance with Doppler echocardiography. Circ Cardiovasc Imaging 2012; 5: 604-612. doi:10.1161/CIRCIMAGING.111.971218

[37] Bollache E, Redheuil A, Clement-Guinaudeau S et al. Automated left ventricular diastolic function evaluation from phase-contrast cardiovascular magnetic resonance and comparison with Doppler echocardiography. J Cardiovasc Magn Reson 2010; 12: 63,429X-12-63. doi:10.1186/ 1532-429X-12-63

[38] Ashrafpoor G, Bollache E, Redheuil A et al. Age-specific changes in left ventricular diastolic function: a velocity-encoded magnetic resonance imaging study. Eur Radiol 2015; 25: 1077-1086. doi:10.1007/s00330014-3488-z

[39] Buss S], Krautz B, Schnackenburg B et al. Classification of diastolic function with phase-contrast cardiac magnetic resonance imaging: validation with echocardiography and age-related reference values. Clin Res Cardiol 2014; 103: 441-450. doi:10.1007/s00392-014-0669-3

[40] Jerosch-Herold M. Quantification of myocardial perfusion by cardiovascular magnetic resonance. J Cardiovasc Magn Reson 2010; 12: 57. doi:10.1186/1532-429X-12-57

[41] Klem I, Heitner JF, Shah DJ et al. Improved detection of coronary artery disease by stress perfusion cardiovascular magnetic resonance with the use of delayed enhancement infarction imaging. J Am Coll Cardiol 2006; 47: 1630-1638. doi:10.1016/j.jacc.2005.10.074

[42] Thomson LE, Fieno DS, Abidov A et al. Added value of rest to stress study for recognition of artifacts in perfusion cardiovascular magnetic resonance. J Cardiovasc Magn Reson 2007; 9: 733-740. doi:10.1080/ 10976640701544415

[43] Zorach B, Shaw PW, Bourque J et al. Quantitative cardiovascular magnetic resonance perfusion imaging identifies reduced flow reserve in microvascular coronary artery disease. J Cardiovasc Magn Reson 2018; 20: 14,018-0435-1. doi:10.1186/s12968-018-0435-1

[44] Kellman P, Hansen MS, Nielles-Vallespin S et al. Myocardial perfusion cardiovascular magnetic resonance: optimized dual sequence and reconstruction for quantification. J Cardiovasc Magn Reson 2017; 19 : 43,017-0355-5. doi:10.1186/s12968-017-0355-5

[45] Watkins S, McGeoch R, Lyne J et al. Validation of magnetic resonance myocardial perfusion imaging with fractional flow reserve for the detection of significant coronary heart disease. Circulation 2009; 120: 2207-2213. doi:10.1161/CIRCULATIONAHA.109.872358

[46] Mordini FE, Haddad T, Hsu LY et al. Diagnostic accuracy of stress perfusion CMR in comparison with quantitative coronary angiography: fully quantitative, semiquantitative, and qualitative assessment. JACC Cardiovasc Imaging 2014; 7: 14-22. doi:10.1016/j.jcmg.2013.08.014

[47] Handayani A, Sijens PE, Lubbers DD et al. Influence of the choice of software package on the outcome of semiquantitative MR myocardial perfusion analysis. Radiology 2013; 266: 759-765. doi:10.1148/ radiol.12120626

[48] van Dijk R, van Assen M, Vliegenthart R et al. Diagnostic performance of semi-quantitative and quantitative stress CMR perfusion analysis: a meta-analysis. J Cardiovasc Magn Reson 2017; 19: 92. doi:10.1186/ s12968-017-0393-z

[49] Kellman P, Hansen MS. T1-mapping in the heart: accuracy and precision. J Cardiovasc Magn Reson 2014; 16: 2,429X-16-2. doi:10.1186/s12968015-0136-y

[50] Reiter G, Reiter C, Krauter C et al. Cardiac magnetic resonance T1 mapping. Part 1: Aspects of acquisition and evaluation. Eur J Radiol 2018; 109: 223-234. doi:10.1016/j.ejrad.2018.10.011

[51] Kim PK, Hong YJ, Im DJ et al. Myocardial T1 and T2 Mapping: Techniques and Clinical Applications. Korean J Radiol 2017; 18: 113-131. doi:10.3348/kjr.2017.18.1.113

[52] Lota AS, Gatehouse PD, Mohiaddin RH. T2 mapping and T2* imaging in heart failure. Heart Fail Rev 2017; 22: 431-440. doi:10.1007/s10741017-9616-5

[53] Reiter U, Reiter C, Krauter C et al. Cardiac magnetic resonance T1 mapping. Part 2: Diagnostic potential and applications. Eur J Radiol 2018; 109: 235-247. doi:10.1016/j.ejrad.2018.10.013

[54] Kellman P, Wilson JR, Xue $\mathrm{H}$ et al. Extracellular volume fraction mapping in the myocardium, part 1: evaluation of an automated method. J Cardiovasc Magn Reson 2012; 14: 63,429X-14-63. doi:10.1186/1532429X-14-63

[55] Ferreira VM, Schulz-Menger J, Holmvang G et al. Cardiovascular Magnetic Resonance in Nonischemic Myocardial Inflammation: Expert Recommendations. J Am Coll Cardiol 2018; 72: 3158-3176. doi:10.1016/ j.jacc.2018.09.072

[56] Diao KY, Yang ZG, Xu HY et al. Histologic validation of myocardial fibrosis measured by T1 mapping: a systematic review and meta-analysis. J Cardiovasc Magn Reson 2016; 18: 92,016-0313-7. doi:10.1186/s12968016-0313-7

[57] Piechnik SK, Ferreira VM, Lewandowski AJ et al. Normal variation of magnetic resonance $\mathrm{T} 1$ relaxation times in the human population at 1.5 T using ShMOLLI. J Cardiovasc Magn Reson 2013; 15: 13. doi:10.1186/1532-429X-15-13

[58] Kellman P, Bandettini WP, Mancini C et al. Characterization of myocardial T1-mapping bias caused by intramyocardial fat in inversion recovery and saturation recovery techniques. J Cardiovasc Magn Reson 2015; 17 : 33. doi:10.1186/s12968-015-0136-y

[59] Wood JC. Cardiac iron across different transfusion-dependent diseases. Blood Rev 2008; 22 (Suppl. 2): S14-S21. doi:10.1016/S0268-960X (08)70004-3

[60] Carpenter JP, He T, Kirk P et al. Calibration of myocardial T2 and T1 against iron concentration. J Cardiovasc Magn Reson 2014; 16: 62,0140062-4. doi:10.1186/s12968-014-0062-4

[61] Kritsaneepaiboon S, Ina N, Chotsampancharoen T et al. The relationship between myocardial and hepatic T2 and $\mathrm{T}^{*}$ at $1.5 \mathrm{~T}$ and $3 \mathrm{~T} \mathrm{MRI} \mathrm{in}$ normal and iron-overloaded patients. Acta Radiol 2018; 59: 355-362. doi: $10.1177 / 0284185117715285$ 